\title{
PREDICTING THE EFFECTS OF DISABILITY GLARE ON DRIVING PERFORMANCE
}

\author{
Rob Gray \\ Department of Applied Psychology \\ Arizona State University \\ Mesa, AZ, USA \\ E-mail: robgray@asu.edu
}

\begin{abstract}
Summary: A driving simulator was used to investigate the effects of simulated glare from the sun on the execution of left-turns at an intersection. The presence of glare resulted in a significant reduction in the safety margin used by drivers (by $0.65 \mathrm{sec}$ on average). The effect of glare was larger for low-contrast than for highcontrast oncoming vehicles. Older drivers (45-60 years) had a significantly greater reduction in safety margin compared to younger drivers (19-29 years), however, there was large inter-driver variability in both age groups. Older drivers adopted a larger safety margin in non-glare conditions with the result that the net effect of glare on driving safety was the same for both age groups. It is proposed that the reduction in retinal image contrast caused by glare caused drivers to overestimate the time to collision with oncoming cars.
\end{abstract}

\section{INTRODUCTION}

When driving in high-glare conditions such as low sun or approaching headlamps at night, the visibilities of objects are reduced and low-contrast objects may be rendered invisible (Miller \& Benede, 1973). A major cause of the problems associated with glare is that light is scattered within the eye onto the retina, thus reducing the contrast of the retinal image (Miller \& Benede, 1973; Van den Berg, 1986). This reduction of contrast is called disability glare. Most drivers are aware that approaching headlights or a low sun can make it more difficult to see objects outside their vehicle and some even avoid high-glare situations (Wolbarsht, 1977). Although it has been suggested that glare susceptibility testing might be used to identify drivers who are especially disabled by glare (Wolbarsht, 1977; Regan, 1991; Babizhayev, 2003), a causal connection between laboratory measures of an individual's glare susceptibility and the probability for accidents in high-glare conditions is lacking. It is even the case that the extent to which glare affects driving performance is not known: "Glare problems are often discussed as a serious threat to the safety of older drivers. However, no studies have demonstrated that increased disability glare is independently associated with either crash involvement or self-reported difficulty in driving (pg. 21)" (Babizhayev, 2003). These are important gaps in our knowledge, given that a high level of glare susceptibility is common in older individuals (Allen, 1967; Regan, 1991), especially in those with early cataract (Miller \& Benede, 1973; Regan, Giaschi \& Fresco, 1993) and that it is projected that the proportion of drivers over the age of 65 will continue to increase over time to roughly 1 in 5 by the year 2030 (NHTSA, 2006). Furthermore, it has also been shown that there is a large individual variation within both old and young age groups to the extent that some young adults are more visually disabled by glare than some much older individuals (Regan, 1991). Therefore, a greater understanding of glare disability could improve road safety for drivers of all ages. 
In the present study, we used a driving simulator to measure the effects of simulated glare from the sun on driving performance. Instead of using a passive task that is only indirectly related to driving (e.g., Schumann et al., 1997), we examined the effects of glare on a complex and dangerous driving maneuver: the execution of a left-turn at an intersection. Of the total of 9.7 million two-vehicle crashes in the USA during the year 2004, approximately $11 \%$ involved a left-turning vehicle (NHTSA, 2006). In Europe, it has been reported that left-turn accidents account for roughly $15 \%$ of total accidents (Larsen \& Kines, 2002). Consistent with this accident data, we have previously shown that drivers are often inaccurate when judging whether there is sufficient time to make a left-turn in front of an oncoming vehicle even under optimal viewing conditions (Gray, 2006). Here we report that the presence of glare increased the frequency of these misjudgments and the incidence of collisions.

\section{METHODS}

\section{Purpose}

As illustrated in Figure 1, judging that a left-turn can be completed safely requires a driver to subtract the estimated time required to complete the turn (TRT) from the estimated time to collision (TTC) with the oncoming vehicle (the safety margin). A safe left turn requires that the driver initiate the turn either when TTC is comfortably larger than TRT, or wait until the oncoming vehicle has passed through the intersection. We varied the speed and initial distance of the oncoming vehicle to present drivers with different values of TTC-TRT ranging from positive (i.e., turn can be completed safely in front of the oncoming car) to negative. Because it has been shown that glare susceptibility depends on the contrast of the test stimulus, we used both a lowand high-contrast oncoming vehicle.

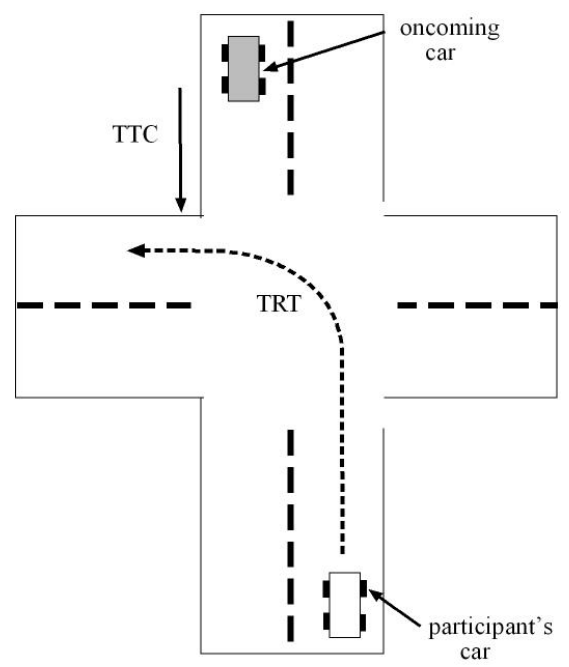

Figure 1. Calculation of safety margin for left-turns. The time required for the driver to complete a left-turn (TRT) was calculated from pilot observations in the driving simulator. This value was then compared to the time required for the oncoming car to reach the middle of the intersection (TTC) 


\section{Apparatus \& Procedure}

Driving Performance. We used a fixed-base driving simulator composed of two main components: (i) a steering wheel mounted on a table top and pedals (Wingman Formula Force GP, Logitech ${ }^{\mathrm{TM}}$ ) and (ii) a 70-deg horizontal X 52-deg vertical display of a simulated driving scene. The visual scene was rendered and updated by DriveSafety ${ }^{\mathrm{TM}}$ driving simulator software running on two PC's (Dell Optiplex GX270). It was projected onto a wall $2.4 \mathrm{~m}$ in front of the participant using a LCD projector (Hitachi CPX1200SER) and was updated at a rate of $60 \mathrm{~Hz}$ in correspondence with the movement of the simulated car.

We simulated a rural 2-lane driving environment. Each trial involved a $4400 \mathrm{~m}$ simulated drive. Speed limit signs of $55 \mathrm{mph}$ were posted throughout the drive and participants were instructed to obey the speed limit at all times. The roadway was made up of five 4-way intersections with traffic lights and one T-junction intersection with traffic lights connected with straight road segments. The participants' instructions were to make a left-hand turn at each of the 4-way intersections and a right-hand turn at the T-junction. Traffic lights were always green as the participant's car approached the intersection. At most of the intersections there was an oncoming car. The initial distance and speed of the approaching cars was varied to create different values of TTC-TRT. Oncoming cars were either dark grey (with a contrast of $9 \%$ relative to the grey road) or white (with a contrast of 54\% relative to the road). The value of TRT for each participant was determined as follows. Before the experimental trials began we had each participant complete 10 practice left-turns with no oncoming cars. TRT was defined as the time between when the steering movement began and when the rear bumper of the participant's vehicle was completely through the intersection. For each participant we used the mean TRT of these 10 practice trials.

We simulated glare from the sun by using a large sheet of glass $(0.9 \mathrm{~m}$ horizontal x $0.9 \mathrm{~m}$ vertical) that was oriented at $45 \mathrm{deg}$ to the participant's line of sight. This glass was used to superimpose a $0.8 \mathrm{deg}$ diameter light source on the driving scene at a height of 20 deg above the simulated road. The luminance of the light source was $120,000 \mathrm{~cd} / \mathrm{m}^{2}$. In the no-glare conditions the light source was turned off. To allow for direct comparison with the VA data, all observers wore an eye patch over their non-dominant eye while driving. The without-glare and with-glare driving conditions each comprised 12 trials for a total of 60 left turns per condition. We used a total of 8 values of TTC-TRT ranging from 3.0 to $-3.0 \mathrm{sec}$. Each of these 8 values was repeated three times for the low-contrast oncoming car and three times for the high-contrast oncoming car. There were also 12 left turns in which there was no oncoming car. Immediately before each driving session, participants completed 3 practice trials.

Glare Questionnaire. In conjunction with the driving simulator experiment described above, we also collected data using a questionnaire designed to assess driver's perceptions about the severity of glare and about how they deal with glare when it is encountered. In this questionnaire participants were asked to rate how much glare affected their driving performance (on a scale from 1-5, with 5 being the most severe) and indicate which methods they used for dealing with glare (e.g., slowing down). 


\section{Participants}

For the driving simulator portion of the study we collected data from participants in two age groups: a group ranging in age from 19 to 29 years and a group ranging in age from 45 to 60 years. All participants held a valid driver's license. The mean number of years of driving experience was 7.3 for the 19-29 group and 32.7 for 45-60 group. All participants were naïve to the aims of the study and were paid an hourly rate. Questionnaire data was collected on a group of 50 participants that did not participate in the driving simulator portion of the study.

\section{Data Analysis}

Driving simulator data were analyzed in separate 2 × 2 × 2 Mixed-factor ANOVA's with age group, contrast and glare presence as factors.

\section{RESULTS}

\section{Driving Performance}

Shown in Figure 2 are the mean left-turn safety margins (i.e., TTC-TRT at the instant of initiating a left turn) for each participant in each of the four conditions. We found that drivers initiated left turns at a significantly smaller safety margin when glare was present $(\mathrm{M}=1.16 \mathrm{sec}$, $\mathrm{SE}=0.05)$ as compared to when the glare source was switched off $(\mathrm{M}=1.81 \mathrm{sec}, \mathrm{SE}=0.07)$ : $[F(1$, $22)=202.9, p<0.001, f=0.59]$. We found a significant main effect of car contrast $[F(1,22)=18.9$, $p<0.001, f=0.43$ ] as left-turns were initiated at a lower value of TTC-TRT for the low-contrast $\operatorname{car}(\mathrm{M}=1.32 \mathrm{sec}, \mathrm{SE}=0.08)$ than for the high-contrast car $(\mathrm{M}=1.65 \mathrm{sec}, \mathrm{SE}=0.07)$. The effect of glare was also significantly larger for the low-contrast oncoming car than for the high-contrast oncoming car, as revealed by a significant glare $\mathrm{x}$ contrast interaction $[F(1,22)=18.9, p<0.001$, $f=0.58]$. It should be noted that under all glare conditions the mean value of TTC-TRT was greater than zero. The significance of this finding is discussed later.

There was also a significant age $\mathrm{x}$ glare interaction $[\mathrm{F}(1,22)=11.4, \mathrm{p}<0.01, f=0.46]$ as the older drivers in our study were more affected by the presence of glare than younger drivers. This last statistic, however, merits more careful examination. When the approaching car was of lowcontrast and the glare source was on, drivers in the 19-29 years age group initiated left turns in 37 out of a possible 144 cases with a negative value of TTC-TRT compared with 12/144 for the with-glare/ high-contrast car situation. (A negative value of TTC-TRT would, in everyday driving, likely result in a collision). When there was no glare these ratios were only $3 / 144$ and 1/144, respectively. For the 45-60 years age group there were a total of 35/144 left-turn initiations of this type for the low-contrast car in the with-glare situation and 19/144 for the high contrast car. When there was no glare these ratios were only $1 / 144$ and $0 / 144$ respectively. Thus, the presence of glare appeared to have a larger effect on the older drivers in our study only because they adopted a more conservative driving strategy in the no-glare conditions. In particular, the mean value of TTC-TRT at the instant of initiating a left turn in the no- glare conditions was significantly lower for the 19-29 years group ( $M=1.7 \mathrm{sec})$ than for the 45-60 years old $(\mathrm{M}=2.0 \mathrm{sec})$ : $\mathrm{t}(11)=1.9, \mathrm{p}<0.05$, though the mean value of TTC-TRT in the with-glare 
conditions was not significantly different for the 19-29 years old ( $\mathrm{M}=1.12 \mathrm{sec})$ and the 45-60 years old age groups $(\mathrm{M}=1.09 \mathrm{sec})$.

\section{Questionnaire}

The average rating for how much glare affected driving performance was $2.7 / 5$. Not surprisingly, the highest ratings were for sunrise (3.4) and sunset (3.6). The sun was cited as the most frequently encountered glare source $(24 \%)$, and the most frequent problems caused by the glare were detecting pedestrians $(21 \%)$ and detecting oncoming traffic (19\%). The most commonly cited methods for combating glare were reducing the frequency of lane changes $(20 \%)$ and avoiding freeways $(24 \%)$. Very few drivers cited leaving bigger gaps $(8 \%)$ or reducing driver speed $(9 \%)$ as ways to reduce the effects of glare on driving.

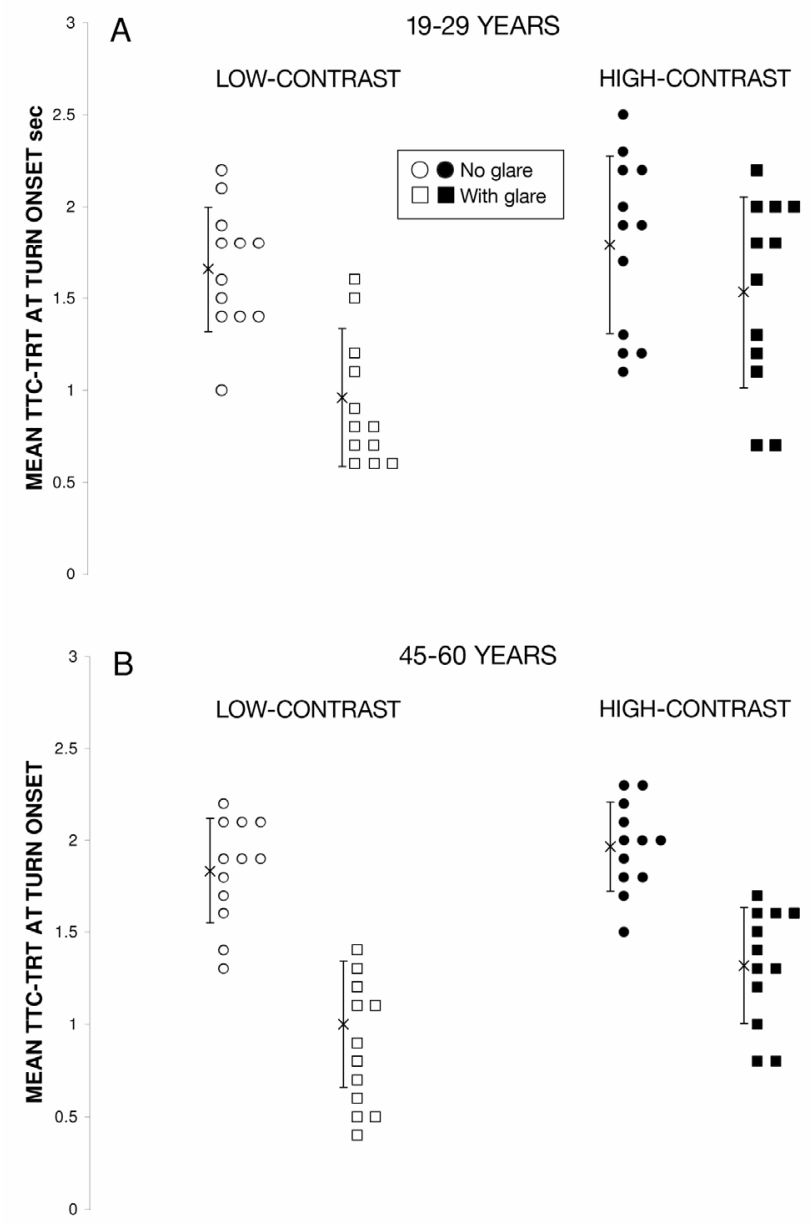

Figure 2. Mean left-turn safety margin (i.e., TTC-TRT) in the glare and no-glare conditions for low- and high-contrast oncoming vehicles. A: 19-29 years age group. B: 45-60 years age group. Crosses and error bars show the mean and standard deviation for each condition 


\section{CONCLUSIONS}

Although the possibility that disability glare may degrade driving performance and be a cause of driving accidents has been discussed in several previous studies, a causal link has not been demonstrated in previous research. Here we provide the first direct evidence that simulated glare from the sun results in an increase in the frequency of unsafe driving maneuvers. Drivers in our study executed left-turns with a significantly smaller margin for error in the presence of glare. On average, the safety margin for left-turn execution was reduced by $0.65 \mathrm{sec}$ when glare was present. In addition, when glare was present there were significantly more turns initiated at a negative value of TTC-TRT, which would in everyday driving likely result in a collision.

Why did glare reduce the safety margin for left-turn execution? First consider the possibility that our drivers did not see the oncoming car in the glare conditions. Suppose that the angle between the simulated sun and the approaching car had been less than the 20-deg value that we used such that the increased intensity of scattered light falling on the fovea was sufficient to render the lowcontrast approaching car invisible for the drivers who were most susceptible to glare. In this case, they would have initiated a left turn independently of the value of TTC-TRT for lowcontrast cars. If that were so, the mean value of TTC-TRT at the initiation of the left turn would be the mean of the stimulus set, i.e., zero. But Figure 2 shows that for every driver, the mean value of TTC-TRT at the onset of the turn was positive, thus rejecting the "invisible car" explanations. So we must look elsewhere to explain the reduction of drivers' safety margin.

We assume that the presence of simulated sun did not induce recklessness in both age groups. In particular, we assume that any given driver's internal criterion of the value of TTC-TRT below which it was not safe to initiate a left turn was the same in both with-glare and no-glare situations, but that in the with-glare situation, and especially for the low-contrast car, the driver's visual system encoded an erroneously low value of TTC. A retinal image correlate of TTC is given in equation (1)

$$
\mathrm{TTC} \approx \frac{\theta}{\mathrm{d} \theta / \mathrm{dt}}
$$

(provided that $\theta$ is small), where $\theta$ is the angular subtense of the approaching object (Hoyle, 1957; Lee, 1976). It has been shown that the rate of retinal image expansion required to evoke a just-detectable sensation of motion in depth is very high for an approaching object whose retinal image contrast is just above contrast detection threshold (i.e., a just-visible object), and that sensitivity to motion in depth increases as the contrast of the retinal image is increased until the contrast of the retinal image is approximately four times contrast detection threshold, at which point sensitivity to motion in depth has increased by approximately 2.7 times (Gray \& Regan, 1996). On this basis we suggest that in the with-glare condition, the reduction of retinal image contrast caused by light scattered onto the fovea, though insufficient to render the approaching car invisible, nevertheless did weaken the motion-in-depth signal, thus causing drivers to overestimate the TTC with the approaching car and hence execute left turns with a reduced safety margin.

On average the value of TTC-TRT at the instant of initiating the left turn was reduced by $0.8 \mathrm{sec}$ for the older drivers in our study as compared to a reduction of $0.5 \mathrm{sec}$ for the younger drivers in our study. This finding is consistent with the fact that, on average, light scattering within the eye 
is more severe in older than in younger age groups (Nadler, Miller \& Nadler, 1990; Regan, 1991). However the results of the present study also clearly indicate that disabling effects of glare should not solely be treated as an "older driver problem." Although on average the effects of glare on driving performance were larger for the older drivers in our study, there was a large inter-subject variability. For the low-contrast oncoming car, 3/12 of the older drivers in our study showed very little effect of glare on driving performance, while 4/12 of the younger drivers showed relatively large effects of glare. Another point that should be emphasized is that because the younger drivers in our study adopted a smaller safety margin in non-glare conditions than older drivers, the smaller effect of glare observed for younger drivers resulted in the same net effect on driving safety as the larger effect of glare observed for older drivers.

During the year 2004, 38,253 people were killed and 1,862,000 injured on the highways of the USA alone (NHTSA, 2006). Although high-glare conditions produced by a low sun comprise only a relatively small proportion of the total time that most of us drive, they tend to occur during times of high traffic density (e.g., during "rush-hour" in the morning and evening). Therefore, reducing accidents caused by glare disability could save a substantial number of lives and prevent a large number of injuries (Babizhayev, 2002). The present study represents a first attempt to directly quantify the effects of glare on driving performance.

\section{ACKNOWLEDGMENTS}

This work was supported by the National Science Foundation Faculty Early Career Development Program (Award \# 0239657 to author R.G.).

\section{REFERENCES}

Babizhayev, M.A. (2003). Glare disability and driving safety. Ophthalmic Research, 35, 19-25.

Elliott, D.B. \& Bullimore, M.A. (1993). Assessing the reliability discriminative ability and validity of disability glare tests. Investigative Ophthalmology \& Visual Science, 34(1), 108119.

Gray, R. (2005). The use and misuse of visual information for "go/no-go" decisions in driving. Hennessy, D. \& Wiesenthal, D. (Eds.). Contemporary Issues in Traffic Research and Road User Safety. New York: Nova Science Publishers, 125-133.

Gray, R. \& Regan, D. (1996). Cyclopean motion perception produced by oscillations of size, disparity and location. Vision Research, 36, 655-666.

Hoyle, F. (1957). The Black Cloud. London: Penguin Books, 26-27.

Lee, D.N. (1976). A theory of visual control of braking based on information about time-tocollision. Perception, 5, 437-459.

Miller, D. \& Benede, K. G. (1973). Intraocular Light Scattering. Springfield, IL: C.C. Thomas.

National Highway Traffic Safety Administration: Traffic Safety Facts Annual Report 2004. Washington, DC, US Department of Transportation, 2006.

Larsen, L. \& Kines, P. (2002). Multidisciplinary in-depth investigations of head-on and left- turn road collisions. Accident Analysis and Prevention, 34, 367-380. 
Regan, D. (1991). The Charles F. Prentice Award Lecture 1990: Specific tests and specific blindness: keys, locks, and parallel processing. Optometry \& Vision Science, 68, 489-512.

Regan, D., Giaschi, D.E., \& Fresco, B.B. (1993). Measurement of glare sensitivity cataract patients using low-contrast letter charts. Ophthalmic Physiol Opt, 13 (2), 115-123.

Schumann, J., M. J. Flannagan, M. J., Sivak, M. \& Traube, E. C. (1997). Daytime veiling glare and driver visual performance: Influence of windshield rake angle and dashboard reflectance. Journal of Safety Research, 28, 133-146.

Van den Berg, T.J.T.P. (1991). On the relation between glare and straylight. Documentra Ophthalmologica, 78, 177-181.

Wolbarsht, M. L. (1977). Tests for glare sensitivity and peripheral vision in driver applicants. Journal of Safety Research, 9, 128-139. 